Port Acadie

Revue interdisciplinaire en études acadiennes

An Interdisciplinary Review in Acadian Studies

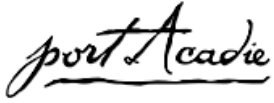

\title{
Le déménagement de la population de Plaisance à l'île Royale
}

\section{Nicolas Landry}

Numéro 18-19, automne 2010, printemps 2011

URI : https://id.erudit.org/iderudit/1010304ar

DOI : https://doi.org/10.7202/1010304ar

Aller au sommaire du numéro

Éditeur(s)

Université Sainte-Anne

ISSN

1498-7651 (imprimé)

1916-7334 (numérique)

Découvrir la revue

Citer cet article

Landry, N. (2010). Le déménagement de la population de Plaisance à l'île Royale. Port Acadie, (18-19), 149-161. https://doi.org/10.7202/1010304ar

\section{Résumé de l'article}

Ce travail vise à présenter un document important pour l'histoire de la fondation de la colonie française de l'île Royale (Cap-Breton) en 1714. Il s'agit d'un mémoire rédigé par le gouverneur de Plaisance, Philippe Pastour de Costebelle, dans le but d'informer le Ministère de la Marine de France sur les ressources de l'île Royale et sur les emplacements envisageables pour y aménager un port et des fortifications. 


\title{
Le déménagement de la population de Plaisance à l’île Royale ${ }^{1}$
}

\author{
Nicolas Landry \\ Université de Moncton (campus \\ de Shippagan)
}

\begin{abstract}
Résumé
Ce travail vise à présenter un document important pour l'histoire de la fondation de la colonie française de l'île Royale (Cap-Breton) en 1714. Il s'agit d'un mémoire rédigé par le gouverneur de Plaisance, Philippe Pastour de Costebelle, dans le but d'informer le Ministère de la Marine de France sur les ressources de l'île Royale et sur les emplacements envisageables pour y aménager un port et des fortifications.
\end{abstract}

\section{Contexte historique}

C'est le 2 septembre 1713 que la France prend officiellement possession du Cap-Breton. Par le fait même, le comte de Pontchartrain, ministre de la marine, donne l'ordre à un contingent d'identifier le meilleur port ou havre pour la nouvelle colonie. Bien que ce choix ne dût pas négliger les considérations stratégiques militaires et commerciales, c'est d'abord en vertu d'accommoder les activités de pêche que le site devait être sélectionné. Le choix de Louisbourg, ou Havre-à-l'Anglais, est arrêté après une visite de tous les autres havres de l'île. Néanmoins, deux autres ports de l'île reçoivent de bonnes notes, soit Port-Toulouse (Saint Peters) et PortDauphin (Englishtown) ${ }^{2}$.

L'historiographie canadienne retient que c'est Nicolas Denys qui est responsable de la première tentative sérieuse d'établissement européen sur l'île du Cap-Breton entre 1650 et 1670. En 1653, détenteur d'un titre officiel, il s'installe à Saint-Pierre (Saint Peters). C'est alors un emplacement stratégique pour la traite avec les Micmacs puisqu'il se trouve sur une route de portage entre l'Acadie et l'arrière-pays du Cap-Breton, dans le voisinage du lac Bras d'Or. C'est Denys lui-même, dans ses écrits, qui mentionne que l'île est depuis longtemps fréquentée par des pêcheurs de La Rochelle. Bien avant Costebelle, auteur du mémoire à l'étude dans ce

1. Philippe Pastour de Costebelle au ministre, 9 juillet 1713, Archives nationales du Canada, MG1, Série C11C, vol. 7, folios 200-208.

2. A. J. B. Johnston, « From port de pêche to ville fortifiée : The Evolution of Urban Louisbourg, 1713-1758 », Aspects of Louisbourg, dans Eric Krause, Carol Corbin, William O'Shea [dir.], University College of Cape-Breton, Sydney, 1995, p. 3-18. 
texte, Denys rend compte des ressources naturelles de l'île et de ses nombreux havres ${ }^{3}$. Il y avait bien eu l'épisode de la tentative ratée de James Stewart ou Lord Ochiltree en 1629 à Port-à-la-Baleine, suite à l'attaque du Français Charles Daniel de Dieppe, qui établit lui-aussi un poste minuscule à Sainte-Anne, emplacement qui vivote jusqu'en $1641^{4}$.

Mais par la suite, Andrew Hill Clark est d'avis que, jusqu'au $\mathrm{xVIII}^{\mathrm{e}}$ siècle, aucune nation ne semble aspirer à s'installer formellement au Cap-Breton et l'île est visitée régulièrement et librement par les navires de pêche, autant d'Europe que des colonies d'Amérique du Nord. Mais en 1706, Jacques Raudot, alors intendant à Québec, suggère un établissement de la France sur l'île et ce, pour trois raisons : servir d'entrepôt entre la France, le Canada, Plaisance et l'Acadie, où les cargaisons pourraient transiter; servir de lieu de pêche d'automne et d'été, incluant l'exploitation de la baleine et des phoques; et, enfin, servir de havre refuge pour les navires marchands et militaires pouvant servir comme base d'opérations maritimes contre les Anglais ${ }^{5}$. Une fois que la France semble décidée à s'installer au Cap-Breton, une vision d'établissement émerge : le développement agricole de l'île Saint-Jean et si possible du Cap-Breton, l'installation de colons en provenance de Plaisance 6 , de l'Acadie, de la nouvelle et de la vieille France, l'exploitation du bois et des mines, la fortification d'un ou de plusieurs ports, l'aménagement d'une base navale et d'un entrepôt pour le commerce. Bref, rien de bien différent de ce que suggérait Raudot en 1706. La France n'avait alors plus le choix de bouger puisqu'en octobre 1714 tous les colons de Plaisance, à l'exception de ceux ayant choisi de prêter le serment de fidélité à la reine Anne, furent transférés aux alentours de Louisbourg, environ 180 personnes, ou à Baleine et à l'île de Scatarie.

Dans ce document, le corsaire bien connu Pierre Maisonnat dit Baptiste fournit au gouverneur Phillipe Pastour de Costebelle de Plaisance, une foule d'informations sur l'île du Cap-Breton?. À son tour, Costebelle renseigne la Cour sur maints aspects à considérer pour mieux planifier

3. Andrew Hill Clark, Acadia: The Geography of Early Nova Scotia to 1760 , The University of Wisconsin Press, Madison, Milwaukee, and London, 1968, p. 264-266.

4. A. J. B. Johnston, L'été de 1744 : La vie quotidienne à Louisbourg au XVIII siècle, Parcs Canada, Ottawa, 1983, p. 11-12.

5. Clark, Acadia... op.cit, p. 268.

6. Pour Plaisance, voir Nicolas Landry, Plaisance Terre-Neuve 1650-1713-Une colonie française en Amérique, Sillery, Septentrion, 2008.

7. W. Austin Squires, "Maisonnat, dit Baptiste, Pierre », Dictionnaire biographique du Canada en ligne, vol. 2 (1701-1740). Capitaine et corsaire, Maisonnat est né en France en 1663. Vers 1693, il est marié et réside à Port-Royal. Mais on sait qu'il demeure à Beaubassin en 1714. Entre 1692 et 1714, il capture plusieurs navires anglais entre Port-Royal et Plaisance. 
le nouvel établissement ${ }^{8}$ : dans un premier temps, quel serait le lieu le plus convenable pour se fortifier dans la nouvelle colonie de la France en Amérique; dans un deuxième temps, l'espoir que les jeunes Acadiens de Nouvelle-Écosse s'établiront sur l'île et que plusieurs deviendront de bons ouvriers qui sauront utiliser les bois, le plâtre et la pierre du pays. Dans un autre ordre d'idée, le document présente plusieurs données sur les ressources naturelles, dont bien sûr la pêche, mais aussi par rapport au potentiel agricole, minier et forestier. Bien entendu, on insiste sur l'importance d'envoyer quelques vaisseaux du roi pour ravitailler la colonie et amener des cadeaux pour maintenir l'alliance des Micmacs.

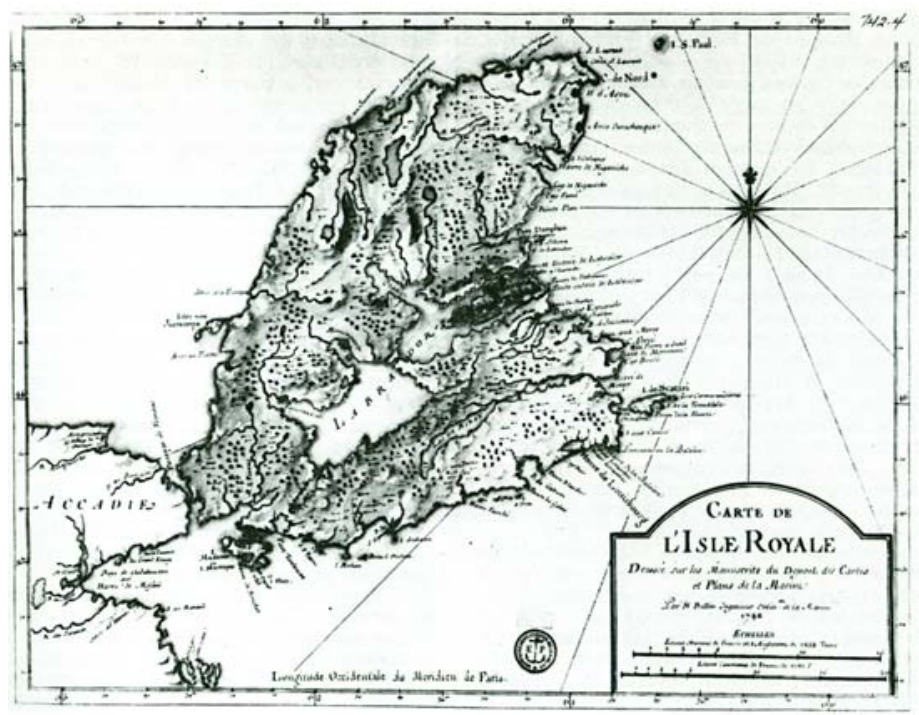

Figure 2. "Carte de I'Isle Royale". Remarque: la majorité des noms de lieu et des établissements se trouvent le long de la côte atlantique de l'île. (Collection de cartes, Forteresse de Louisbourg, no 1742-4.)

Source : B.A. Balcom, La pêche à la morue à l'Île Royale, 1713-1758, op. cit., p. 11.

8. Georges Carbelaud Salagnac, «Pastour de Costebelle, Philippe », DBC en ligne, vol. 2. Officier, gouverneur de Plaisance, puis de l'île Royale, né en 1661 en France, mort en 1717 à Port-Dauphin (Englishtown, Nouvelle-Écosse). Nommé lieutenant d'infanterie en 1687, il arrive à Plaisance la même année avec 25 soldats. Il doit attendre jusqu'en 1706 pour obtenir le poste de gouverneur et, en 1708, est nommé Chevalier de Saint-Louis. Durant la première année d'occupation de l'île Royale, il doit s'assurer du logement de 160 personnes au cours de l'hiver. Ce n'est qu'à l'été de 1714 qu'il en termine avec l'évacuation de Plaisance. De 1713 à 1716, la population de l'île Royale passe à environ 3000 personnes. 
"Tout ce qui peut contribuer au bien et à l'établissement de cette nouvelle colonie».

Depuis que votre grandeur ma fait l'honneur de me donner avis par ses lettres de la présente année en date du 24 février et 13 mars que le roi avait formé le projet d'établir les habitants de la colonie de Plaisance sur l'île du Cap Breton, je me suis appliqué autant qu'il m'a été possible de tirer tous les éclaircissements convenables aux desseins de sa majesté et découvrir par une exacte recherche les personnes capables de m'instruire sur ce sujet.

Une de celles que j'ai trouvé la mieux informée de la situation de la dite île est un ancien habitant de la côte d'Acadie nommé Baptiste Maisonnat, expérimenté dans la navigation et qui a parcouru la plus grande partie des ports et baies attenantes à cette côte. Desquelles il m'a fait la démonstration par une carte quoique irrégulière qui ne laisse pas de donner une idée très instructive de la situation des dits ports et baies, de leur profondeur, des dangers de leur entrée, de la bonté des terres qui y sont contiguës, des côtes les plus abondantes en poisson pour la pêche des morues et de celles où elle peut se pratiquer la plus avantageusement, tant pour la sécherie que pour la sûreté des chaloupes occupées dans ce commerce.

Après l'avoir examiné sans prétendre rien décider de mon propre choix, il m'a paru qu'il n'y avait aucun port dans cette île dont l'entrée fut plus aisée à fortifier que celle du havre à l'Anglais ${ }^{9}$ qui est dans le centre de la côte du sud, à une lieue ou deux du havre à la baleine entre la baie de Scatary et celle de Gabarous, dont l'une est abondante en bois de construction et mature et l'enceinte de celle de Scatary toute entourée de prairie dans un plat pays où il n'y a pas de chemin du fond du havre à l'Anglais pour aller par la terre à l'une et à l'autre plus d'une lieue et demie et fort aisé de pratiquer des routes à faire rouler les chariots pour entretenir par ce moyen une communication utile pour les nécessités des voitures ${ }^{10}$.

Dans ledit havre à l'Anglais d'une étendue suffisante à mouiller en sûreté au moins le nombre de 40 à 50 navires et y pratiquer une sécherie tant en grave que vigneaux ou claies pour la pêcherie de 20 vaisseaux en laissant des places vacantes pour plusieurs habitants du côté de bâbord, et ce

9. Havre-à-l'Anglais est le site de Louisbourg.

10. L'île de Scatary est située sur la côte est du Cap-Breton, au nord de Louisbourg, alors que la baie de Gabarouse est au sud de Louisbourg. 
qu'il y a à observer de plus avantageux pour la sûreté de la navigation des chaloupes en pêche est qu'étant forcé par les gros vents de relâcher, il y a des ports de retraite sur toute la côte. Ce qui ne se trouve point sur celle qui gît depuis le Cap Breton jusqu'au cap de nord et par ainsi on ne pourrait y pêcher qu'avec des sloops ou brigantins. À quoi nos pêcheurs ne sont pas accoutumés comme les Anglais de la côte de Boston, qui au lieu des chaloupes biscayennes ne se servent que des sloops sur les bancs de l'Acadie où ils tiennent toujours le large et ne rapportent à terre que lorsqu'ils ont leur charge de morues ${ }^{11}$.

Après avoir entretenu votre grandeur sur l'importance de cet article qui est un des plus à observer dans un établissement de cette nature, je reviendrai s'il lui plaît à l'avantage du lieu pour les fortifications du port à l'Anglais qui ne se trouve dans aucun autre de l'île y ayant sur la pointe qui avance à tribord de son entrée un terrain élevé qui n'est point commandé et aussi spacieux qu'il le faut pour étendre les fortifications convenables à la sûreté de lad entrée. Du côté de bâbord il y a un îlot à pouvoir y construire une bonne redoute et des batteries qui se trouveront à la pointe du fort qui est celle qui forme sa largeur. Il y a de plus une base cachée sous l'eau qui est difficile à éviter pour les navigateurs qui ne sont pas prévenus de ses dangers.

Cette situation avantageuse pour la pêche et pour la défense du port est sans contredit préférable à toutes les autres baies beaucoup plus profondes et spacieuses, qui sont celles des Espagnols, l'Indienne, de Scatary, de Gabarous et de Sainte-Anne, du côté du nord dans laquelle on peut mouiller un plus grand nombre de vaisseaux et trouver une plus grande étendue de terrain que dans le havre à l'Anglais. Mais dans aucune on ne saurait établir le commerce de la pêche qu'avec des sloops ou brigantins comme j'ai eu l'honneur de le présenter à votre grandeur ci-devant, ni s'y fortifier avantageusement que par des travaux et des dépenses immenses. Ce qu'on évitera dans ledit havre à l'Anglais, situé sur la côte la plus abondante en poisson et voisin d'une lieue et demi de distance par mer et par terre des baies de Scatary et de Gabarous de même que du havre à la baleine dans lequel

11. Pour des descriptions des embarcations de pêche de la Nouvelle-Angleterre au xVIII ${ }^{\mathrm{e}}$ siècle, voir B. A. Balcom, La pêche de la morue à l'île Royale, 1713-1758, Ottawa, Parcs Canada, 1984. Pour les conflits résultant de la présence des pêcheurs de la Nouvelle-Angleterre le long des côtes acadiennes avant 1710, voir Robert Guitard, "Le déclin de la Compagnie de la pêche sédentaire en Acadie de 1697 à 1702 ", Cahiers de la Société historique acadienne, vol. 9, n¹, mars 1978, p. 5-21. 
4 à 5 navires peuvent établir leur pêche et leur sécherie. Et depuis ledit port à la baleine jusqu'aux îles Michau tirant à l'ouest, il peut s'établir tout le long de la côte des degrats et échaffaux pour un très grand nombre de vaisseaux ${ }^{12}$.

J'ai jusqu'à présent entretenu votre grandeur des difficultés et des commodités différentes qui peuvent se pratiquer pour le commerce de la pêche et lui ai donné les éclaircissements de la différence qu'il y a à faire de la côte du nord à celle du sud. Ainsi je n'aurai pas avoir rien a y augmenter mais pour ne rien laisser à lui dire sur tout ce qui peut contribuer au bien et à l'établissement de cette nouvelle colonie. Je crois devoir lui rapporter tout ce qui peut se pratiquer d'avantageux pour les terres qui consiste à établir des maîtres constructeurs pour toutes sortes de bâtiments propres à entretenir la navigation. Lesdits anciens habitants français des côtes de l'Acadie ${ }^{13}$ et du Canada sont naturellement fort adroits à manier la hache et industrieux pour toutes sortes d'ouvrages. Particulièrement pour mettre en siage les bois propres à la construction, soit pour le gain ou pour l'envie de se perfectionner dans ce métier. La plupart des jeunes gens nés dans cette partie de l'Amérique accoutumée à vivre sous la domination française ne manqueront pas de se jeter parmi les peuples de leur nation et en très peu de temps il se formera nombre de bons ouvriers dans le pays qui, dans la suite, pourront fournir à des travaux de grande utilité pour l'agrandissement du pays. D'autant plus que l'île du Cap Breton produit toutes sortes de bois convenables à l'usage de la Marine tant pour la construction que pour la mature dont je cru devoir donner le dénombrement.

Conformément au juste rapport qui m'en a été fait, savoir bois de chêne, bois de frêne, de hêtre, d'érable, de tremble, de sapin, d'épinette, de bouleau, de merisier, de plaine, dont je ne connais pas la qualité que l'on m'a dit être doux et pliant approchant de l'érable. À cela il faudra ajouter de bonnes forges et moulins à scie, le charbon de terre y est abondant en différents endroits, le plâtre de même. Je n'ai point pu savoir au juste s'il y a des terres bonnes à faire de la brique, n’y de la pierre à chaux, mais je ne doute pas qu'il ne s'y trouve de l'un et de l'autre. Ce qu'il y a d'important et

12. Tous ces ports de pêche sont situés sur la côte est du Cap-Breton, dans le voisinage de Louisbourg ou de l'île Madame.

13. Sur la présence des Acadiens à l’île Royale, voir Bernard Pothier, "Les Acadiens à l'île Royale, 1713-1734 ", Cahiers de la Société historique acadienne, $23^{\mathrm{e}}$ cahier, 1969, p. 96-111. Voir plus récemment A. J. B Johnston, "Un regard neuf sur les Acadiens de l'île Royale », Cahiers de la Société historique acadienne, vol. 33, n³, septembre 2002, p. 142-155. 
d'avantageux pour l'établissement et qui facilitera beaucoup les ouvrages des fortifications ${ }^{14}$. C'est toute la côte du nord qui est bordée d'une carrière presque générale d'une pierre blanche et tendre, aisée à tailler et couper de la grosseur qu'on pourra le souhaiter.

Par la présence des sauvages, le blé d'Inde y vient dans les terres parfaitement en maturité de même que les citrouilles. Sans difficulté, toutes sortes de graines y produiront une bonne récolte et on y élèvera sans peine et sans une grosse dépense des bestiaux de toutes les espèces. Voilà mon seigneur ce qui est de source certaine. Je lui envoi ci-joint la carte générale de l'île pour démonstration seulement, n'étant tirée qu'à l'idée du nommé Baptiste Maisonnat bon navigateur et bien instruit de ce que j'ai l'honneur de lui rapporter.

Le sieur Baptiste Maisonnat se trouvant à Plaisance au retour de l'île du Cap Breton où il a été contraint d'hiverner dans le port à la baleine, les glaces l'ayant surpris dans sa route venant de Beaubassin côte d'Acadie. Dans ce port le mois de janvier dernier je n'ai cru pouvoir rien faire de plus utile au service du roi dans la conjoncture présente que de le retenir jusqu'à l'arrivée du navire le Semslack commandé par Monsieur de Saint-Ovide ${ }^{15}$ et lui ordonne de s'embarquer comme une personne expérimentée dans le pilotage et bon connaisseur de tous les ports de ladite île du Cap-Breton et qui de plus parle la langue des sauvages. Par le moyen de laquelle on peut tirer de grands éclaircissements pour la connaissance des ports de ladite île du Cap-Breton. Je lui ai promis que Votre grandeur reconnaîtrait ses services de la manière qu'il pourrait le souhaiter. Depuis le jour que je l'ai arrêté je lui ai fais fournir son entretient des magasins du roi, de même qu'à quatre hommes de l'équipage qui sont à ses gages pour

14. Sur la question des fortifications de Louisbourg, voir Frederic Thorpe, Remparts lointains : la politique française des travaux publics à Terre-Neuve et à l'île Royale, 1695-1758, Ottawa, Éditions de l'Université d'Ottawa, 1980.

15. Bernard Pothier, "Monbeton de Brouillan, dit Saint-Ovide, Joseph de ", $D B C$ en ligne, vol. 3 (1741-1770). Officier dans les troupes de la Marine, gouverneur de l'île Royale, né en France en 1676, décédé en 1755. Arrivé à Plaisance en 1692 avec son oncle Jacques-François de Monbeton en qualité d'enseigne dans les troupes de la Marine, il est promu lieutenant en 1694 puis capitaine en 1696. C'est à Plaisance qu'il accomplit les plus hauts faits d'armes de sa carrière en Amérique. Il obtient le grade de lieutenant du roi vers 1706 et se signale tout particulièrement dans la prise de St.John's en 1709. Suite au traité d'Utrecht, il reçoit le commandement de la flûte Semslack afin de mener à bien l'évacuation de Plaisance. En 1714, il est promu lieutenant de vaisseau, remplit la charge d'administrateur de la colonie à compter de 1716, avant d'être confirmé comme gouverneur au printemps 1718. 
naviguer le bâtiment sur lequel il devait s'en retourner à Beaubassin dans son ancienne habitation où réside sa femme et ses enfants qu'il prétend retirer de la domination anglaise et se rétablir sous le gouvernement de son légitime souverain ayant fidèlement servi sa majesté pendant tout le temps de la dernière guerre même avec beaucoup de distinction par les fréquentes et continuelles courses qu'il a fait sur les ennemis de l'état étant un digne sujet pour la guerre et pour tout ce qui pu se pratiquer d'utile dans le métier des hommes de mer ${ }^{16}$.

Par cette juste et avantageuse relation, je suis persuadé que Votre grandeur répandra sur lui toutes les grâces qu'il peut raisonnablement espérer dans l'état présent de sa fortune, et je lui ai promis d'en faire ressouvenir votre grandeur dans l'occasion. Permettez-moi au surplus Monseigneur de lui répéter ce que j'ai eu l'honneur d'exposer dans les mémoires que j'ai donné à Votre grandeur l'année dernière des côtes et ports de cette partie de l'Amérique qui conviennent dans l'entreprise d'un nouveau établissement.

Il ne faut rien épargner pour la solidité des fortifications et qu'il n'y faut rien commencer qu'on ne soit bien en état de finir et conduire à la perfection. Mais pour parvenir à y réussir il faut observer en premier lieu d'ordonner des fonds suffisants, à ne pas contraindre les entrepreneurs de surseoir les ouvrages.

Votre grandeur qui n'ignore pas qu'un officier peut être d'un mérite distingué et d'une valeur égale sans avoir toute l'habilité requise dans le génie bien qu'il soit sur les rangs des personnes qui possèdent cette science conviendra que la longue pratique dans ce métier instruit beaucoup plus que la théorie et que plusieurs prennent le nom d'ingénieur et qui ne sont que des copistes et incapables de construire par leurs propres lumières des ouvrages convenables à la difficulté d'un terrain qu'on leur ordonne d'occuper ${ }^{17}$.

Ainsi, soit par de trop grandes ou trop faibles idées après avoir employé tout leur savoir faire et consommé des

16. Au sujet de la situation des Acadiens au début du régime anglais en NouvelleÉcosse, voir John G. Reid et al., The Conquest of Acadia, 1710 - Imperial, Colonial, and Aboriginal Constructions, Toronto, University of Toronto Press, 2004 et Geoffrey Plank, An unsettled Conquest - The British Campaign Against The People of Acadia, Philadelphia, University of Pennsylvania Press, 2001.

17. Il est fort probable que Costebelle fait allusion à Jacques L'Hermitte, ingénieur à Plaisance et avec qui il a souvent eu des divergences de vues sur la marche à suivre dans les travaux de fortifications. L'Hermitte fut ingénieur, officier d'état-major et cartographe, ingénieur et major de la garnison de Plaisance de 1695 à 1714, souslieutenant de roi et ingénieur à l'île Royale en 1714 et 1715. Dictionnaire biographique du Canada en ligne, vol. 2, 1701-1740. 
fonds considérables, il ne font jamais rien qui vaille. Ces sortes de fautes sont difficiles à réparer et l'on y parvient qu'avec des grosses dépenses sans y comprendre à la longueur du temps ce qu'on paie et qu'on doit éviter dans le commencement de tous les projets par le choix d'un second Monsieur Vauban, s'il s'en trouvait encore en France.

Mais sans faire cette recherche n'y insulter à la capacité de Monsieur Lhermitte que j'estime véritablement. Votre grandeur peut sans rien craindre pour le Canada envoyer un ordre à Monsieur de Beaucours ${ }^{18}$ de se rendre à l'île du CapBreton comme ingénieur général de la Nouvelle-France et d'y faire jeter les fondements des fortifications praticables et convenables aux terrains que les troupes doivent occuper, de même que pour les logements. Si l'avis que j'ai l'honneur de lui donner sur ce sujet est du goût de Votre grandeur elle aura la bonté de m'en informer par les premiers vaisseaux des ports de France auxquels on expédiera des passeports pour le voyage de Plaisance et d'y joindre les ordres qu'elle juge à propos pour ledit Sieur de Beaucours. Je ne manquerai point d'occasion pour lui faire remettre en diligence non plus qu'il [s'en trouve?] pour se rendre de Québec au Cap-Breton avant même que cette garnison y soit arrivée.

Après ledit fort construit sur l'île du Cap-Breton il est de conséquence de recourir aux moyens qui peuvent procurer quelque douceur aux habitants et faciliter ceux de pourvoir à faire rejaillir le même avantage sur les troupes du roi destinées pour y tenir garnison et ce qui me paraît praticable par les expédients que je vais proposer à Votre grandeur, qui consistent à faire une levée de trois en trois une de 60 à 80 jeunes gens depuis l'âge de 16 jusqu'à 18 $\& 20$ ans enrôlés comme soldats et venus dans la colonie par les ordres du gouverneur du pays. Pour les dépenses au service des habitants pendant l'espace de 3 ans sans être habillés ni nourris aux dépens de Sa majesté, c'est-à-dire que les habitants chez lesquels on les mettra pour ainsi dire à l'école pour l'espace desdites trois années seront obligés de rembourser au trésorier du roi servant dans le pays.

Les avances qui auront été faites par Sa majesté pour la levée desdits engagés de même que les hardes et vivres

18. Josué Dubois Berthelot de Beaucours (1662-1750) fut officier dans les troupes de la Marine, ingénieur en chef du Canada, gouverneur de Trois-Rivières et de Montréal. Le 10 mars 1715, Beaucours est assigné au poste d'ingénieur de l'île Royale. Pourtant, dès l'année suivante, on nomme Jean-François de Verville directeur des fortifications de l'île Royale et Beaucours est muté de Port-Dauphin (Englishtown) à Port-Toulouse (Saint Peters). Dictionnaire biographique du Canada en ligne, vol. 3, 1741-1779. 
fournis pour leur passage. Moyennant quoi lesdits habitants seront en droit de les employer pendant l'espace de 3 ans à tous les travaux convenables au commerce qu'ils pratiqueront tant pour la pêche que pour la culture des terres et les fonds avancés pour cette dépense seront comptés en recette audit trésorier sur ceux qui devront être remis audit pays et pour les ouvrages des fortifications. En quoi sa Majesté donnera un secours considérable pour l'agrandissement de cette nouvelle colonie et à la fin desdites trois années de service parmi lesdits habitants, ils rentreront dans les compagnies de la garnison pour y faire les fonctions de soldats. Ce qui servira pour ainsi dire de pépinière pour les recrues des troupes et fournira des bons hommes exercés aux travaux et fatigues du pays et qui dans toutes sortes d'ouvrages pourront être employés utilement pour les services du roi et facilitera les moyens aux capitaines d'infanterie de donner des congés d'ancienneté sans affaiblir la garnison qui pendant les premières années n'étant pas d'un grand secours. Des nouvelles recrues de France n'étant le plus souvent composées que des enfants et qui avant qu'ils soient formés au métier de la guerre sont plusieurs années d'une médiocre utilité. Ce qui sera corrigé par l'expédiant ci-dessus dont les habitants et les troupes recevront un gros avantage n'étant point obligés de donner d'autres salaires à ces jeunes gens que celui de l'entretient, des vivres et habits jusqu'au temps expiré desdites trois années. Après lesquelles ayant été élevés soit à cultiver les terres soit au commerce de la pêche, la garnison ne manquera jamais de travailleurs et de bons soldats et les anciens congédiés exercés dès leur jeunesse à tout ce qui peut se pratiquer d'avantageux pour leur établissement pendant le reste de leur vie, peupleront l'île d'hommes laborieux et endurcis au climat du pays froid et rude à supporter aux nations élevées dans l'Europe ${ }^{19}$.

Votre grandeur doit aisément prévoir que lorsque des peuples habitués dans un pays depuis plusieurs années sont contraints par l'ordre de leur souverain d'abandonner leurs maisons et d'interrompre leur commerce qui est l'unique ressource qui contribue à les faire subsister avec leurs familles et qu'il faut dans ce changement, faire un trajet de mer avec tous les embarras d'un ménage se trouvent en ce cas la plupart exposés dans une misère et une extrémité bien difficile à supporter. Voilà mon seigneur la situation prochaine des habitants de Plaisance qui malgré tous ces contretemps

19. Transformer des soldats en habitants suite à leurs années de service faisait alors partie de la stratégie de peuplement français en Amérique. 
sont tous infiniment disposés à sacrifier leur biens et leur vie pour aller continuer leurs services et leurs travaux où le roi l'ordonnera.

Mais en pareille occasion, Votre grandeur aura la bonté de réfléchir que ce peuple était destiné pour être transplanté sur des terres où l'on ne peut subsister sans des secours étrangers pendant les deux premières années de leur établissement. Il ne suffira pas seulement de leur faire transporter des vivres mais qu'il faut encore que l'autorité de Sa majesté agisse dans les ports de France pour que les marchands qui auront ordinairement pour le commerce de la pêche des morues un ordre positif d'entretenir le même commerce qu'à Plaisance en sel, agrès et autres nécessités dont ils sont parfaitement informés. Sans quoi tous lesdits peuples habitants de la nouvelle colonie périront de froid et de faim. Dégradés sur une île déserte et malgré toutes ces précautions, je prévois que si Sa majesté ne répand des grâces particulières en envoyant de ses propres vaisseaux chargés de vivres et de sel qui est l'article principal pour faire fleurir le commerce du pays, cette dite nouvelle colonie ne saurait se soutenir les premières années de son établissement ${ }^{20}$.

Tout au contraire le roi faisant les premières avances de ses fonds dans d'autres intérêts que le remboursement du capital, le pays se rendra bon et fertile par les travaux de ses habitants qui dans la suite y entretiendront un commerce beaucoup plus avantageux que celui qui s'est pratiqué jusqu'à présent dans l'île de Terre-Neuve. Il ne faut pas négliger de faire établir la nation sauvage des missions françaises des côtes de l'Acadie, du côté du passage de Canso et leur faire des villages pour les entretenir dans notre parti et leur continuant les dons gratuits ordinaires. Sans quoi ils pourraient probablement se laisser entraîner aux largesses des Anglais ${ }^{21}$. Il faut [illisible] solidement sur cet article étant

20. Kenneth Donovan aborde la question des incitatifs offerts aux anciens colons de Plaisance et aux Acadiens pour les convaincre de s'installer à l'île Royale. Bien que les habitants de Plaisance et de l'Acadie se voient offrir des concessions de terre gratuites, les Acadiens veulent également être certains d'être en mesure de trouver des terres cultivables. C'est ce qui explique leur préférence pour Port-Dauphin. Mais en fin de compte, seulement 67 familles émigrent au Cap-Breton entre 1713 et 1734. Néanmoins, certaines d'entre elles retourneront en Acadie. Kenneth Donovan, "Communities and Families : Family Life and Living Conditions in Eighteenth-Century Louisbourg ", dans Aspects of Louisbourg, p. 117-149. Toujours au sujet de la problématique acadienne et de leur déplacement au Cap-Breton, voir également A. J. B. Jonston, "D’autres serments de loyauté en Acadie ", Cahiers de la Société historique acadienne, vol. 33, $\mathrm{n}^{\circ}$ 3, septembre 2002, p. 142-155.

21. Sur toute la question des présents nécessaires au maintien de l'alliance avec la 
d'une grande importance pour le présent et pour l'avenir d'attirer et d'attacher ces nations dans nos intérêts.

Je ne discontinue pas Monseigneur de faire des vœux pour la santé et prospérité de Votre grandeur et salut avec un très profond respect.

Plaisance, le 9 juillet 1713

Votre humble et très obéissant serviteur

De Costebelle.

\section{Conclusion}

La démarche de la France de coloniser le Cap-Breton suite à la perte de Terre-Neuve et de l'Acadie n'est alors pas une décision prise au hasard. Alors qu'il était en poste à Plaisance, l'ingénieur et major Jacques L'Hermitte reçoit l'ordre, en 1698, de visiter toutes les côtes et rades de l'Acadie et de la baie des Chaleurs. En 1714, à nouveau, on lui demande de refaire le même exercice. Mais les directives semblent ambiguës. Bien que l'on semble convaincu que les Acadiens de la Nouvelle-Écosse se rendront à l'île Royale, on précise qu'il ne faudra pas les empêcher pour autant de déménager à l'île Saint-Jean ou à la baie des Chaleurs. Pourtant, la préférence envers l'île Royale apparaît plus évidente dans une correspondance du ministre de la Marine au père Justinien alors qu'on l'avise clairement de convaincre les Acadiens de choisir l'île Royale. Selon le ministre, l'île Royale regorge " de bonnes terres, des plus beaux bois du monde, de bons pâturages d'excellent foin à proximité de la baie de Brador $»^{22}$. On sait que la qualité des terres fut nettement contestée par une délégation d'Acadiens qui les inspectèrent. Ce qui explique en partie pourquoi à peine une soixantaine de familles s'y rendirent à l'invitation des autorités françaises. Cependant, il est probable que cette inspection se limita aux terres longeant les côtes puisque d'autres beaucoup plus fertiles existaient dans

nation micmaque, voir deux auteurs importants : Olive Patricia Dickason, " La guerre navale des Micmacs contre les Britanniques, 1713-1763 ", dans Charles A. Martijn [dir.], Les Micmacs et la mer, Montréal, Recherches amérindiennes au Québec, 1986, p. 233-248; et William C. Wicken, Mi'kmaq Treaties on Trial: History, Land, and Donald Marshall Junior, Toronto, University of Toronto Press, 2002.

22. Le ministre à Jacques L'Hermitte, 26 mars 1698, Bibliothèque et Archives Canada, MG1-B, vol. 20, f 53-55, microfilm C3755. Le ministre à L'Hermitte, 21 mars 1714, BAC-MG1-B, vol. 36, f 39-41, microfilm $C_{3763}$. Le ministre au père Justinien, 23 mars 1714, BAC-MG1-B, vol. 36, f 447-48, microfilm C3762, 3763. Il s'agit du père Justinien Durand, prêtre, récollet, missionnaire, né en France vers 1667 et décédé à Québec en 1746. Il est arrivé à Port-Royal en 1704 avec les titres de grand vicaire de l'évêque de Québec et de curé de Port-Royal. Après le traité d'Utrecht en 1713, à la demande du gouvernement français, lui et d'autres missionnaires essaient d'inciter la population acadienne à émigrer dans les territoires demeurés français. Dictionnaire biographique du Canada en ligne, vol. 3, 1741-1779. 
l'arrière-pays de l'île Royale et furent d'ailleurs exploitées avec succès par les immigrants écossais à compter de la fin du xvIII siècle ${ }^{23}$.

23. Voir à ce sujet Rusty Bitterman, «The Hierarchy of the Soil : Land and Labour in a 19th Century Cape Breton Community », Acadiensis, vol. xvIII, n 1, automne 1998, p. 33-55. 